\title{
Review: lasting health behaviour changes began with critical self appraisal and small steps that led to an identity shift
}

Kearney MH, O'Sullivan J. Identity shifts as turning points in health behavior change. West J Nurs Res 2003;25:134-52.

What are the key influences and processes that enable people to make lasting health behaviour changes (eg, in diet, exercise, smoking cessation, and substance abuse recovery)?

\section{DATA SOURCES}

Studies were identified by searching CINAHL, Medline, Sociofile, and PsycLIT and by hand searching relevant journals (to 2000).

\section{STUDY SELECTION AND ASSESSMENT}

Articles or dissertations were selected if they were qualitative reports, addressed turning points or a process of change in health behaviour over time, and were grounded in or supported by observational or verbatim qualitative data. 2 reviewers independently coded study findings in categories of main concept, pre-change influences, turning point, and steps in the change process. Categories were refined through discussion as inductive coding and analysis unfolded.

\section{MAIN FINDINGS}

14 studies (399 participants, 73\% women; 6 studies on weight loss and cardiovascular risk reduction, 6 on substance abuse recovery, and 2 on smoking cessation) were included. Distressing accumulated evidence. The process of health behaviour change began with awareness of a growing body of distressing evidence that was incongruent with personal goals and values (eg, memory lapses, auto accidents, or loss of custody of children for substance abusers). Conflict with longstanding values or goals prompted self appraisal, described as taking a hard look at myself. This critical self appraisal, not the specific crisis, was the turning point that initiated behaviour change. Constraints that might interfere with critical self appraisal included the need for stability in life, perceptual impairment, accommodating personal norms, insufficient time to stop for self appraisal, social isolation, and social pressure to maintain the status quo. One small step. The important step in launching behaviour changes was a small, tentative first effort that, if successful, enabled further change. Noticeable rewards of the first small change helped people believe that continued change was possible. If the small step failed, people would either seek another approach to change or readjust to accommodate the current status quo. Seeing myself in a new light. The ability to take on a new personal and social self helped sustain behaviour changes and occurred gradually. Insight into motivating and sustaining the change helped people shift their thinking. Walking the walk, talking the talk. People added new For correspondence: Dr M H Kearney, William F Connell School of Nursing, Boston College, Chestnut Hill, MA, USA. kearnema@bc.edu Source of funding: no external funding. behaviours to help sustain behavioural changes and used self talk to maintain their focus on the desired behaviour and goals. Avoiding the undesired behaviour often required assertiveness that was new to their identity. Taking control of their environment was important for promoting change.

\section{CONCLUSION}

Making a behaviour change that lasted $\geqslant 6$ months involved a critical reappraisal of self and situation, which was the first turning point that led to small change. If this small change was successful, it produced positive indicators of a possible new identity, which led to more behavioural changes over time.

\section{Commentary}

K earney and O'Sullivan's investigation is the only grounded formal theory study of health behaviour change across problem behaviours (ie, diet, smoking, and substance use). This work is important because it presents a provisional model for understanding how behaviour patterns can become more health promoting

Grounded formal theory is a type of research synthesis that is intended to explain processes and refine theory in order to improve clinical outcomes, research, and healthcare policy. ${ }^{1}$ A potential hazard for such syntheses is overgeneralisation of findings such that they are of little practical use. ${ }^{2}$ However, this does not appear to be the case in this study, although failure to discuss contradictory findings among studies might suggest that differences between change processes have been obfuscated. The strengths of this work include a pair of researchers working together to enhance interpretive balance, the use of evidentiary quotations, and development of theory grounded in evidence.

The results offer potentially important insights into how change occurs across various behaviour patterns associated with ill health. It is suggested that despite the underlying problem, similarities in the change process are striking, and interventions to alter hazardous health related behaviours may be similar. Nurses are encouraged to promote this process by helping individuals assess their longstanding values and goals, promoting self confidence, and supporting movement in a positive direction. Nurses should also be aware that barriers can impede progress, and individuals may require support to deal with intermittent challenges. Additional research is needed to further explicate the model and to determine how this time delayed process can be initiated or hastened.

Deborah L Finfgeld, PhD, APRN, BC University of Missouri-Columbia Columbia, Missouri, USA

1 Finfgeld DL. Metasynthesis: the state of the art-so far. Qual Health Res 2003; 13:893-904.

2 Paterson BL, Thorne SE, Canam C, et al. Meta-study of qualitative health research: a practical guide to meta-analysis and meta-synthesis. Thousand Oaks, CA: Sage Publications, 2001. 